\title{
Space-Time Block Coded and IQ-interleaved TCM, TTCM, BICM and BICM-ID Assisted OFDM
}

\author{
S. X. Ng, T. H. Liew and ${ }^{1}$ L. Hanzo \\ Dept. of ECS, University of Southampton, SO17 1BJ, UK. \\ Tel: +44-23-8059 3125, Fax: +44-23-8059 4508 \\ Email: ${ }^{1}$ lh@ecs.soton.ac.uk, http://www-mobile.ecs.soton.ac.uk
}

\begin{abstract}
Space-Time Block Coded (STBC) In-phase Quadrature-phase (IQ)-interleaved Coded Modulation (CM) schemes are proposed for Orthogonal Frequency Division Multiplexing (OFDM). Trellis Coded Modulation (TCM), Turbo TCM (TTCM), Bit-Interleaved Coded Modulation (BICM) and Iteratively Decoded BICM (BICM-ID) schemes having a similar decoding complexity are employed in conjunction with 16-level Quadrature Amplitude Modulation (16QAM) for yielding an effective throughput of 3 information bits per symbol. The IQ-diversity attained by the proposed schemes provides a significant additional coding gain. It was found that TTCM and BICM-ID assisted STBC-IQ-OFDM were the best schemes in terms of the achievable bit error ratio and frame error ratio, respectively.
\end{abstract}

\section{INTRODUCTION}

Recently the family of transmit diversity techniques [1, 2] has been studied extensively for employment at the base station, since it is more practical to have multiple transmit antennas at the base station, than at the mobile station. Space-Time Trellis Coding (STTC) pioneered by Tarokh et. al. [3] incorporates jointly designed channel coding, modulation, transmit diversity and optional receiver diversity [1]. In an attempt to reduce the associated decoding complexity, Alamouti proposed Space-Time Block Coding (STBC) [1, 4] employing two transmit antennas. Alamouti's scheme was later generalised to an arbitrary number of transmit antennas $[1,5]$.

Trellis Coded Modulation (TCM) [1, 6] was originally designed for transmission over Additive White Gaussian Noise (AWGN) channels, where it is capable of achieving a coding gain without bandwidth expansion. Turbo TCM (TTCM) [1,7] is a more recently proposed bandwidth efficient transmission scheme, which has a structure similar to that of the family of binary turbo codes, distinguishing itself by employing TCM schemes as component codes. Both the TCM and TTCM schemes employed set partitioning based signal labelling, in order to increase the minimum Euclidean distance between the encoded information bits. Symbol interleavers were utilised both for the turbo interleaver and for the channel interleaver, for the sake of achieving time diversity when communicating over Rayleigh fading channels.

Another powerful Coded Modulation (CM) scheme utilising bitbased channel interleaving in conjunction with Gray signal labelling, which is referred to as Bit-Interleaved Coded Modulation (BICM), was proposed in [8]. It combines conventional non-systematic convolutional codes with several independent bit interleavers. The number of parallel bit-interleavers used equals the number of channel coded bits in a symbol $[1,8]$. Recently, iteratively decoded BICM using Set Partitioning (SP) based signal labelling, referred to as BICM-ID has also been proposed [9].

In an effort to increase the achievable time diversity, a multidimensional TCM scheme utilising a symbol interleaver and two en- coders was proposed in [10], where the individual encoders generate the In-phase (I) and Quadrature-phase (Q) components of the complex transmitted signal, respectively. Another TCM scheme using constellation rotation was proposed in [11], which utilised two separate channel interleavers for interleaving the I and Q components of the complex transmitted signals, but assumed the absence of I/Q crosscoupling, when communicating over Rayleigh fading channels. Explicitly, I/Q cross-coupling is the effect imposed by the complex channel where the I (or Q) component of the received signal becomes dependent on both the I and Q components of the transmitted signal. Recently, a new approach which amalgamates STBC [4] with IQinterleaved CM (STBC-IQ-CM) schemes using no constellation rotation were proposed for transmission over AWGN and Rayleigh fading channels with the presence of I/Q cross-coupling in [12, 13]. It was shown in [12] that the STBC-IQ-based TCM/TTCM scheme is capable of quadrupling the achievable diversity potential of conventional single-transmitter symbol-interleaved TCM/TTCM, when communicating over narrowband uncorrelated Rayleigh fading channels. This was achieved without degrading their performance, when communicating over AWGN channels. Furthermore, the STBC-IQ-BICM-ID scheme of [13] is also capable of exploiting the IQ diversity owing to employing iterative detection.

In this contribution, the STBC-IQ-CM schemes of [12, 13] are adopted for transmission over dispersive Rayleigh fading channels in the context of an OFDM system [14]. The effect of time- and frequency-domain Rayleigh fading on the proposed schemes was studied and the achievable performance gain was quantified.

\section{SYSTEM OVERVIEW}

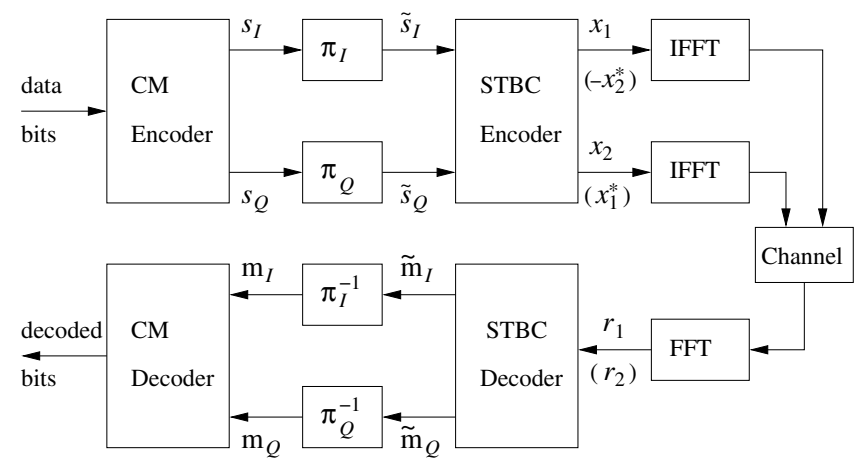

Figure 1: Block diagram of the STBC-based IQ-interleaved CM assisted OFDM system. The notations $\pi$ and $\pi^{-1}$ denote the interleaver and deinterleaver, while (.) denotes the STBC signals during the second symbol period.

The block diagram of the STBC-based IQ-interleaved CM as- 
sisted OFDM system is shown in Figure 1. Specifically, we employ two transmitters and one receiver in the $G_{2}$ STBC $[1,4]$ scheme. Furthermore we invoke two independent IQ interleavers in the CM arrangement, which are denoted as $\pi_{I}$ and $\pi_{Q}$ in the block diagram of Figure 1. More explicitly, the conventional symbol interleaver of TCM/TTCM [1] is substituted by the separate I and Q interleavers. By contrast, the bit interleavers of BICM/BICM-ID are retained and the bit interleaving process is performed inside the CM encoder and decoder blocks. We denote the frequency-domain IQ-interleaved signal by $\tilde{s}=\tilde{s}_{I}+j \tilde{s}_{Q}$, which is encoded by the STBC encoder. The output signals $x_{1}$ and $x_{2}$ of the STBC seen in Figure 1 are mapped to individual Inverse Fast Fourier Transform (IFFT) stages for the sake of OFDM modulation [14]. The corresponding OFDM-modulated signals are then transmitted using two separate antennas over the timedomain dispersive Rayleigh fading channels to the receiver.

At the receiver, a single Fast Fourier Transform (FFT) block is used for demodulating $r_{1}$ and $r_{2}$ arriving during the two received time-slots. When the number of OFDM subcarriers is sufficiently high we may assume that each OFDM subcarrier experiences narrowband, non-dispersive channel conditions. Therefore, the corresponding frequency-domain flat Rayleigh fading channel exhibits a complex fading coefficient of $h=\alpha e^{j \theta}$. As seen in Figure 1, during the first symbol period of the STBC transmission [1, 4], the signals $x_{1}=\tilde{s}_{1}$ and $x_{2}=\tilde{s}_{2}$ are OFDM-modulated and transmitted, while during the second symbol period, the signals $-x_{2}^{*}$ and $x_{1}^{*}$ are OFDM-modulated and emitted from the transmit antennas 1 and 2, respectively. Unfortunately, neither the fading magnitude nor the phase of the complex frequency-domain Rayleigh fading channel transfer function factor is constant across the two STBC time-slots since they both evolve according to the statistics of the frequency-domain Rayleigh fading channels. The signal is also contaminated by the zeromean AWGN $n$ exhibiting a variance of $\sigma^{2}=N_{0} / 2$, where $N_{0}$ is the single-sided noise power spectral density.

However, if we follow Alamouti's proposition [4] and assume that the frequency-domain Rayleigh fading magnitude and phase of the channel transfer function factor are constant across the two STBC time-slots, then we can obtain the estimates of $x_{1}$ and $x_{2}$ from the STBC decoder as [12]:

$$
\left(\begin{array}{l}
\hat{x}_{1} \\
\hat{x}_{2}
\end{array}\right)=\left(\alpha_{1}^{2}+\alpha_{2}^{2}\right)\left(\begin{array}{l}
x_{1} \\
x_{2}
\end{array}\right)+\left(\begin{array}{c}
\hat{n}_{1} \\
\hat{n}_{2}
\end{array}\right),
$$

where $\alpha_{t}, t \in\{1,2\}$ is the frequency-domain Rayleigh fading magnitude between transmit antenna $t$ and the receive antenna, which is assummed to be constant across the two STBC time-slots, while $\hat{n}_{1}$ and $\hat{n}_{2}$ are the corresponding noise components contaminating $x_{1}$ and $x_{2}$. However, when Alamouti's assumption is not satisfied by the channel, there will be interference between the signals of the two transmit antennas, resulting in an residual error floor. Explicitly, the signal $\hat{x}_{1}=\left(\alpha_{1}^{2}+\alpha_{2}^{2}\right) x_{1}+\hat{n}_{1}$ is the decoupled version of $r_{1}$, where $\hat{x}_{1, I}$ (or $\hat{x}_{1, Q}$ ) is independent of $x_{1, Q}$ and $x_{2, Q}$ ( or $x_{1, I}$ and $x_{2, I}$ ).

Hence, it can be readily shown that the associated IQ branch metrics of the STBC coded signal $x_{1}=x_{1, I}+j x_{1, Q}$ can be derived from $\hat{x}_{1}=\hat{x}_{1, I}+j \hat{x}_{1, Q}$ as [12]:

$$
\tilde{\mathrm{m}}_{I}\left(x_{1, I} \mid \hat{x}_{1, I}, D_{I}\right)=-\frac{\left|\hat{x}_{1, I}-D_{I} x_{1, I}\right|^{2}}{2 \sigma^{2} D_{I}}
$$

and

$$
\tilde{\mathrm{m}}_{Q}\left(x_{1, Q} \mid \hat{x}_{1, Q}, D_{Q}\right)=-\frac{\left|\hat{x}_{1, Q}-D_{Q} x_{1, Q}\right|^{2}}{2 \sigma^{2} D_{Q}},
$$

where we have $D_{I}=D_{Q}=D=\left(\alpha_{1}^{2}+\alpha_{2}^{2}\right)$. The corresponding branch metric of $x_{2}$ is computed similarly. The effect of the associated second order transmit diversity attained may be observed in the context of the term $\left(\alpha_{1}^{2}+\alpha_{2}^{2}\right)$.

Note that $\tilde{\mathrm{m}}_{I}$ and $\tilde{\mathrm{m}}_{Q}$ share the same channel-envelope related value of $D=D_{I}=D_{Q}=\left(\alpha_{1}^{2}+\alpha_{2}^{2}\right)$ for the same transmitted signal of $x(=\tilde{s})$, but after the IQ deinterleavers of $\pi_{I}^{-1}$ and $\pi_{Q}^{-1}$ seen in Figure $1, \mathrm{~m}_{I}$ and $\mathrm{m}_{Q}$ will be associated with different $D$ values. The branch metric of the TCM/TTCM-coded signal $s$ is computed from:

$$
\mathrm{m}(s)=\mathrm{m}_{I}\left(x_{I}=s_{I}\right)+\mathrm{m}_{Q}\left(x_{Q}=s_{Q}\right) .
$$

Since there are two independent IQ coordinates for a complex CM symbol, and since they are independently interleaved by the interleavers $\pi_{I}$ and $\pi_{Q}$ in Figure $1, \mathrm{~m}_{I}$ and $\mathrm{m}_{Q}$ provide independent diversity for a particular complex-valued symbol. More explicitly, since we have $D_{I} \neq D_{Q}$ for $\mathrm{m}_{I}$ and $\mathrm{m}_{Q}$, the IQ-interleaved TCM/TTCM scheme is expected to double the achievable diversity order compared to its symbol-interleaved counterpart. Therefore the achievable Hamming distance of the IQ-interleaved TCM/TTCM scheme is based on the number of different I and Q coordinates between the different transmitted messages, rather than on the number of different symbols, which was the case in the context of conventional symbolinterleaved TCM/TTCM. Note that there is no IQ-interleaving gain for the 16QAM-based IQ-BICM scheme, since the I (or Q) interleaver will only further randomise two BICM output bit-sequences in parallel, resulting in two similarly randomised bit-sequences. However, the 16QAM-based BICM-ID scheme is still capable of exploiting the IQ diversity owing to employing iterative detection, but the IQ diversity gain is not as much as that of TCM/TTCM.

\section{SIMULATION RESULTS}

We evaluated the Bit Error Ratio (BER) and Frame Error Ratio (FER) versus signal to noise ratio per bit, namely $E_{b} / N_{0}$, performance of the proposed schemes using 16-level Quadrature Amplitude Modulation (16QAM) in the context of the non-iterative 64-state TCM and BICM schemes. These results were compared to those achieved by the similar-complexity iterative 8-state TTCM arrangement using four decoding iterations and along with an 8-state BICM-ID arrangement using eight decoding iterations. The generator polynomials expressed in octal format for TCM and TTCM are $\left[\begin{array}{llll}101 & 16 & 64 & 0\end{array}\right]$ from [6] and [ $\left.\begin{array}{llll}11 & 2 & 4 & 10\end{array}\right]$ from [7], respectively. BICM and BICMID employ Paaske's non-systematic convolutional codes [15] and their generator polynomials shown in octal format are :

$$
\left[\begin{array}{llll}
6 & 1 & 0 & 7 \\
3 & 4 & 1 & 6 \\
2 & 3 & 7 & 4
\end{array}\right] \text { and }\left[\begin{array}{cccc}
4 & 4 & 4 & 4 \\
0 & 6 & 2 & 4 \\
0 & 2 & 5 & 5
\end{array}\right] \text {, respectively. }
$$

As regards to the associated algorithmic complexity, the rationale of using 64 and 8 trellis states, respectively, was that the TCM/BICM and TTCM/BICM-ID schemes considered here exhibit a similar decoding complexity expressed in terms of the total number of trellis states. Explicitly, since there are two 8-state TTCM decoders, which are invoked in four iterations, we encounter a total of $2 \cdot 8 \cdot 4=64$ TTCM trellis states. By contrast, only a single 8-state BICM-ID decoder is required, which is invoked in eight iterations, involving a total of $8 \cdot 8=64$ BICM-ID trellis states. The effective throughput was 3 Bits Per Symbol (BPS) for all the 16QAM based CM schemes. The equally 3 BPS-throughput STBC aided 8-level Phase-Shift-Keying (8PSK) scheme using no channel coding and communicating over 


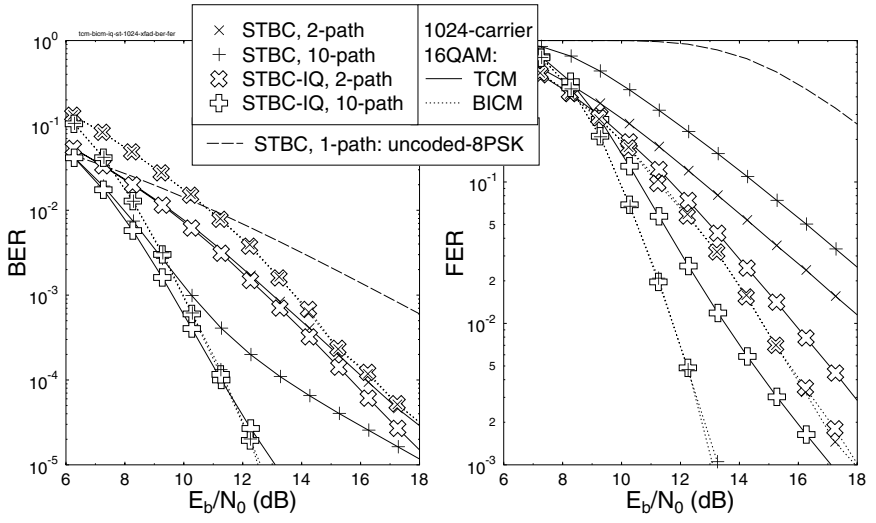

Figure 2: BER and FER versus $E_{b} / N_{0}$ performance of the 16QAM based TCM and BICM assisted 1024-subcarrier 3 BPS effectivethroughput OFDM schemes, when communicating over an uncorrelated dispersive Rayleigh fading channel.

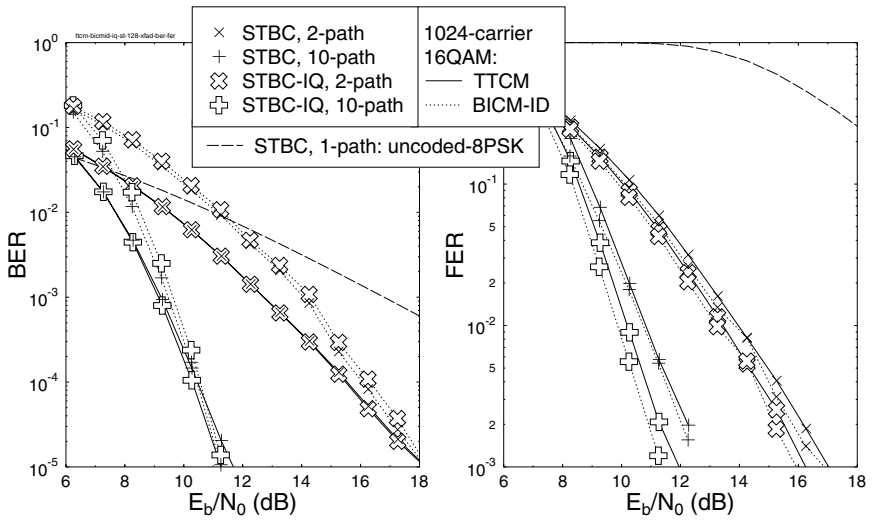

Figure 3: BER and FER versus $E_{b} / N_{0}$ performance of the 16QAM based TTCM and BICM-ID assisted 1024-subcarrier 3 BPS effective-throughput OFDM schemes, when communicating over an uncorrelated dispersive Rayleigh fading channel.

a narrowband Rayleigh fading channel was employed as a benchmarker. A total interleaver delay of 1024 symbol durations, which also corresponses to a codeword length of 1024 symbols, was used for the $\mathrm{CM}$ schemes. All schemes utilised two transmit antennas for the $G_{2}$ STBC [4] and one receiver antenna.

\subsection{Time-domain Uncorrelated Rayleigh Fading}

In this section the performance of the proposed scheme communicating over a Rayleigh fading channel, which was uncorrelated in the time-domain, was evaluated in Figures 2 to 5. Equal-power 2-path and 10-path CIR models having one OFDM sample duration spacing between each of the paths were employed and each path experienced independent Rayleigh fading. Furthermore, OFDM systems having 128 and 1024 subcarriers were employed. More explicitly, one OFDM symbol has a duration of $128+32(1024+256)$ OFDM samples for an 128-subcarrier (1024-subcarrier) OFDM system, where a cyclic prefix of $128 / 4=32(1024 / 4=256)$ samples was employed to overcome the inter-OFDM symbol interference due to the channel's memory.

Let us first study the effect of the frequency-domain Rayleigh fading on the STBC-IQ-CM based OFDM schemes in Figures 2 and 3,

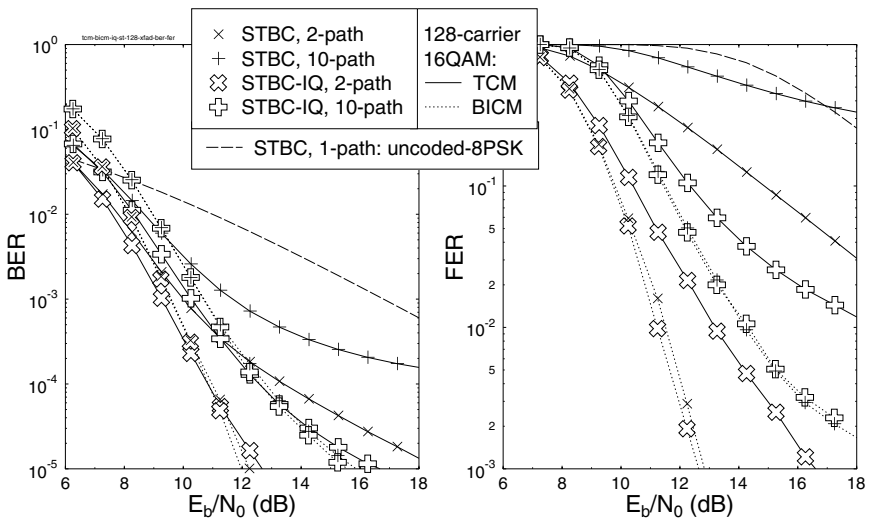

Figure 4: BER and FER versus $E_{b} / N_{0}$ performance of the 16QAM based TCM and BICM assisted 128-subcarrier 3 BPS effectivethroughput OFDM schemes, when communicating over an uncorrelated dispersive Rayleigh fading channel.

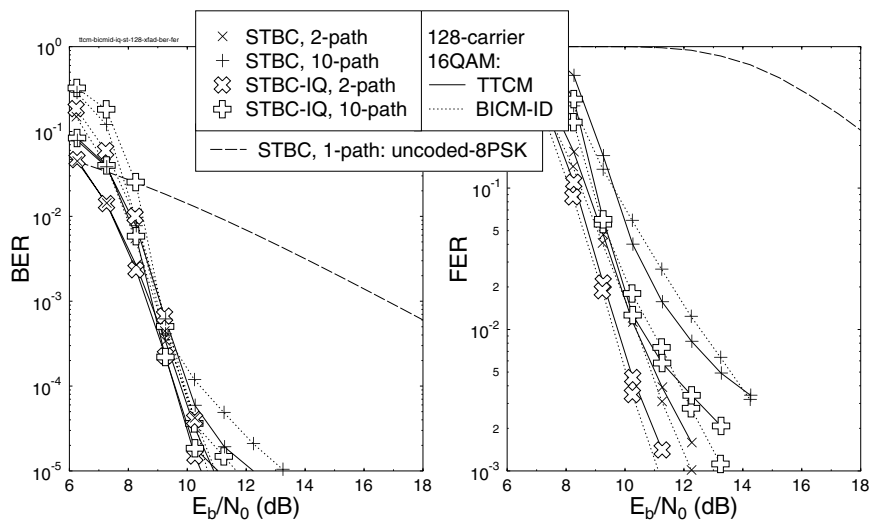

Figure 5: BER and FER versus $E_{b} / N_{0}$ performance of the 16QAM based TTCM and BICM-ID assisted 128-subcarrier 3 BPS effectivethroughput OFDM schemes, when communicating over an uncorrelated dispersive Rayleigh fading channel.

where the number of OFDM subcarriers is 1024. Since the codeword length of the CM schemes is also 1024 symbols, the performance of the schemes depends on the frequency-domain Rayleigh fading. Note that the frequency-domain fading rate of the 2-path CIR model is significantly slower than that of the 10-path CIR model. Futhermore, we note that it has been shown in $[12,13]$ that the IQ-diversity is more beneficial in fast fading narrowband channels than in slow fading narrowband channels. As shown in Figures 2 and 3, a similar performance trend is observed in the context of the OFDM system studied here, when the STBC-IQ-CM schemes are communicating over dispersive Rayleigh fading channels. Explicitly, all STBC-IQ-CMOFDM schemes, except for STBC-IQ-BICM-OFDM, exhibit a higher performance gain than their more conventional STBC-CM-OFDM counterparts, when communicating over the 10-path CIR model compared to the 2-path CIR model.

However, the BER performance of STBC-IQ-BICM-ID-OFDM is marginally worse than that of STBC-BICM-ID-OFDM, although this trend was reversed in terms of the achievable FER, as shown in Figure 3. The marginally worse BER performance of IQ-BICM-ID is attributed to the error propagation phenomenon encountered during its iterative decoding, when a received frame is severely corrupted. 
By contrast, the good FER performance of IQ-BICM-ID is attributed to its excellent iterative decoding gain achieved with the aid of an extra IQ-diversity, when a received frame is moderately corrupted. This may be further interpreted by observing that numerous received frames are completely decontaminated from the transmission errors by iterative detection with the aid of IQ-diversity, which improves the FER. By contrast, badly corrupted frames will suffer from more bit errors after iterative detection, which degrades the BER slightly but not the FER. From Figures 2 and 3, we found that STBC-IQTCM-OFDM exhibits the highest IQ-diversity gain, while STBC-IQTTCM-OFDM and STBC-IQ-BICM-ID-OFDM are the best performers in terms of the BER and FER attained, respectively.

Let us now investigate the effect of both the frequency-domain and time-domain Rayleigh fading on the STBC-IQ-CM based OFDM schemes in Figures 4 and 5, where the number of OFDM subcarriers is 128. Since the codeword length of the CM schemes, is higher than the number of OFDM subcarriers, the CM decoder will wait for eight received frames each having 128 coded symbols, before the decoding process is invoked. When transmitting a 1024-subcarrier OFDM symbol, it is possible that all the subcarriers encounter a deep fade. By contrast, in case of using eight consecutive 128-subcarrier OFDM symbols, the channels are that some of the OFDM symbols are received at a higher than average power, and hence all of its subcarriers experience less hostile fading. This phonomenon is a manifestation of the higher diversity potential associated with the transmission of the shorter, 128-subcarrier OFDM symbol across eight transmission timeslots. Therefore, the performance of the schemes depends on both the velocity of the frequency-domain as well as that of the time-domain Rayleigh fading fluctuation. As a result, the transmitted interleaved codeword hosting 1024 symbols will experience a relatively uncorrelated frequency-domain fading when the OFDM system employs 128 rather than 1024 subcarriers, while communicating over the timedomain uncorrelated Rayleigh fading channel. Note that when communicating over uncorrelated/fast fading channels, the STBC scheme will exhibit a BER/FER error floor, since the fading magnitude and the phase of the complex frequency-domain Rayleigh fading channel transfer function factor are no longer constant across the two STBC time-slots. Owing to this fact, the STBC scheme is expected to suffer a higher performance degradation, when communicating over the 10-path CIR model compared to the 2-path CIR model.

As illustrated in Figures 4 and 5 for the 128-subcarrier OFDM system, the performance of the STBC-IQ-CM or STBC-CM schemes communicating over the 10-path CIR model is worse than that over the 2-path CIR model. Note from Figure 4 that without IQ-diversity, the BER/FER performance of STBC-TCM-OFDM communicating over the 10-path CIR model is worse than that of the STBC-8PSK benchmarker using no channel-coding when communicating over a narrowband channel, at a moderately high $E_{b} / N_{0}$ value. However, STBC-IQ-CM-OFDM still exhibits a higher performance gain, than its conventional STBC-CM-OFDM counterpart, when communicating over the 10-path CIR model compared to the 2-path CIR model in the context of the 128-subcarrier OFDM system. Again, STBCIQ-TTCM-OFDM and STBC-IQ-BICM-ID-OFDM are the best performers in terms of the BER and FER attained, respectively.

\subsection{Time-domain Correlated Rayleigh Fading}

In this section the performance of the proposed scheme communicating over a Rayleigh fading channel which is correlated in timedomain, is evaluated in Figures 6 and 7. An equal-power 2-path CIR model having a delay of $5 \mu \mathrm{s}$ between the paths was employed. Each path experienced independent Rayleigh fading having a maximum

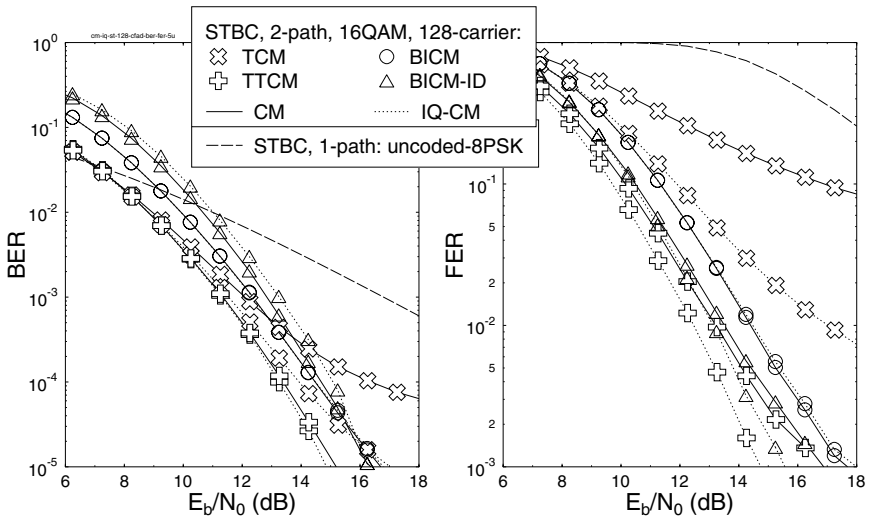

Figure 6: BER and FER versus $E_{b} / N_{0}$ performance of the 16QAM based CM assisted 128-subcarrier 3 BPS effective-throughput OFDM schemes, when communicating over a correlated 2-path Rayleigh fading channel having a normalised Doppler frequency of $2.5 \times 10^{-4}$.

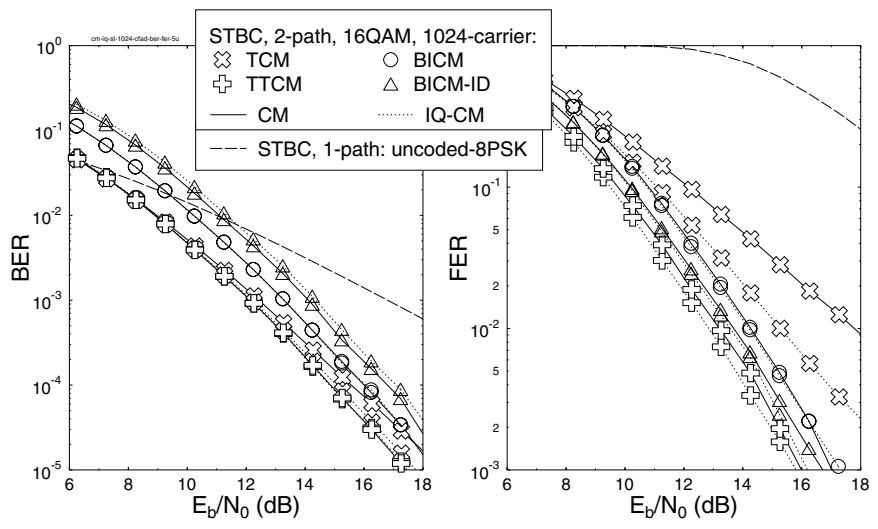

Figure 7: BER and FER versus $E_{b} / N_{0}$ performance of the 16QAM based CM assisted 1024-subcarrier 3 BPS effective-throughput OFDM schemes, when communicating over a correlated 2-path Rayleigh fading channel having a normalised Doppler frequency of $2.5 \times$ $10^{-4}$.

Doppler frequency of $200 \mathrm{~Hz}$. The overall system's effective Baudrate was $800 \mathrm{Ksymbol} / \mathrm{s}$, which corresponded to a time-domain symbol duration of $1.25 \mu \mathrm{s}$. This represents a slowly fading channel having a normalised Doppler frequency of $200 \mathrm{~Hz} \times 1.25 \mu \mathrm{s}=2.5 \times 10^{-4}$. In the 128-subcarrier OFDM system, each OFDM symbol has a duration of $160 \mu \mathrm{s}$ and a cyclic prefix duration of $40 \mu \mathrm{s}$, which corresponds to 128 and 32 symbol durations in the time-domain, respectively, based on the system's effective Baudrate. Similarly, for the 1024-subcarrier OFDM system each OFDM symbol has a duration of $1280 \mu \mathrm{s}$ (1024 symbols) and a cyclic prefix duration of $320 \mu \mathrm{s}$ (256 symbols).

As portrayed in Figures 6 and 7, the IQ-diversity gain of the schemes is more apparent in terms of the FER performance than in terms of the BER performance. With the aid of IQ-CM, the error floor imposed by the impaired-orthogonality STBC decoding is circumvented. Explicitly, when communicating over this dispersive correlated Rayleigh fading channel, STBC-IQ-TTCM-OFDM is the best performer in terms of the achievable BER and FER. Note that at a FER of $5 \times 10^{-3}$, the coding gain of STBC-IQ-TTCM-OFDM over uncoded STBC-8PSK is about $18.8 \mathrm{~dB}$ and $18.2 \mathrm{~dB}$, when employing a 128- and 1024-subcarrier OFDM system, respectively. Again, 
STBC-IQ-TCM exhibits the highest IQ-diversity gain amongst the various STBC-IQ-CM schemes.

\section{CONCLUSIONS}

In this contribution, we have incorporated the STBC-IQ-CM scheme of [12] into an OFDM system for communication over dispersive Rayleigh fading channels. It was shown that STBC-IQ-CM-OFDM provides a significant performance gain, when the joint benefits of transmit-, IQ-, time- and frequency-diversity were exploited simultaneously. When comparing the various CM schemes operating at a similar decoding complexity using a range of different scenarios, the STBC-IQ-TTCM-OFDM and STBC-IQ-BICM-ID-OFDM schemes were found to attain the highest performance in terms of the BER and FER, respectively.

Furthermore, we can draw the following conclusions from Section 3.1 when the proposed schemes were communicating over channels exhibiting uncorrelated/rapid time-domain Rayleigh fading:

- provided that the channel exhibits a high number of propagation paths, it is better to invoke a higher number of OFDM subcarriers in order to avoid the detrimental effects imposed by the impaired-orthogonality STBC decoding,

- if the channel is expected to exhibit a low number of propagation paths, it is better to use a lower number of OFDM subcarriers, so that the time-domain diversity can be efficiently exploited.

When the STBC-IQ-CM-OFDM schemes, except for STBC-IQ-BICMOFDM, were communicating over correlated Rayleigh fading channels having a slow fading rate and exhibiting a 2-path CIR, the IQdiversity gain was eroded, when considering the BER, although still exhibiting an improved FER performance, as discussed in Section 3.2.

\section{ACKNOWLEDGEMENTS}

The financial support of the European Union under the auspices of the SCOUT project and the EPSRC, Swindon UK is gratefully acknowledged.

\section{REFERENCES}

[1] L. Hanzo, T. H. Liew and B. L. Yeap, Turbo Coding, Turbo Equalisation and Space Time Coding for Transmission over Wireless channels. New York, USA: John Willy IEEE Press, 2002.

[2] A. Wittneben, "Base station modulation diversity for digital SIMULCAST," IEEE Vehicular Technology Conference, pp. 505-511, 1993.

[3] V. Tarokh, N. Seshadri and A. R. Calderbank, "Space-time codes for high rate wireless communication: Performance analysis and code construction," IEEE Transactions on Information Theory, vol. 44, pp. 744-765, March 1998.

[4] S. M. Alamouti, "A simple transmitter diversity scheme for wireless communications," IEEE Journal on Selected Areas in Communications, vol. 16, pp. 1451-1458, October 1998.

[5] V. Tarokh, H. Jafarkhani and A.R. Calderbank, "Space-Time Block Codes from Orthogonal Designs," IEEE Transactions on Information Theory, vol. 45, pp. 1456-1467, July 1999.

[6] G. Ungerböck, "Channel coding with multilevel/phase signals," IEEE Transactions on Information Theory, vol. 28, pp. 55-67, January 1982.

[7] P. Robertson, T. Wörz, "Bandwidth-efficient turbo trellis-coded modulation using punctured component codes," IEEE Journal on Selected Areas in Communications, vol. 16, pp. 206-218, February 1998.
[8] E. Zehavi, "8-PSK trellis codes for a Rayleigh fading channel," IEEE Transactions on Communications, vol. 40, pp. 873-883, May 1992.

[9] X. Li, J. A. Ritcey, "Bit-interleaved coded modulation with iterative decoding using soft feedback," IEE Electronics Letters, vol. 34, pp. 942 943, May 1998.

[10] S. Al-Semari and T. Fuja, "I-Q TCM: Reliable communication over the Rayleigh fading channel close to the cuttoff rate," IEEE Transactions on Information Theory, vol. 43, pp. 250-262, January 1997.

[11] B. D. Jelicic and S. Roy, "Design of trellis coded QAM for flat fading and AWGN channels," IEEE Transactions on Vehicular Technology, vol. 44, pp. 192-201, February 1994.

[12] S. X. Ng and L. Hanzo, "Space-Time IQ-interleaved TCM and TTCM for AWGN and Rayleigh Fading Channels," IEE Electronics Letters, vol. 38, pp. 1553-1555, November 2002.

[13] S. X. Ng and L. Hanzo, "Space-Time Block Coded IQ-interleaved Joint Coding and Modulation for AWGN and Rayleigh Fading Channels," in IEEE Vehicular Technology Conference, (Jeju, Korea), 22-25 April 2003.

[14] L. Hanzo, M. Münster, B-J. Choi and T. Keller, OFDM and MC-CDMA for broadband multiuser communications, WLANs and Broadcasting. New York, USA: John Willy IEEE Press, May 2005.

[15] S. Lin and D. J. Costello, Jr, Error Control Coding: Fundamentals and Applications. Inc. Englewood Cliffs, New Jersey 07632: Prentice-Hall, 1983. 